\title{
Permanent postoperative hypoparathyroidism: an analysis of prevalence and predictive factors for adequacy of control in a cohort of 260 patients
}

\author{
Juan J. Díez ${ }^{1,2}$, Emma Anda ${ }^{3}$, Julia Sastre ${ }^{4}$, Begoña Pérez Corral ${ }^{5}$, Cristina Álvarez-Escolá ${ }^{6}$ \\ Laura Manjón ${ }^{7}$, Miguel Paja $^{8}$, Marcel Sambo ${ }^{9}$, Piedad Santiago Fernández $^{10,11}$, \\ Concepción Blanco Carrera ${ }^{12}$, Juan C. Galofré ${ }^{13}$, Elena Navarro ${ }^{14}$, Carles Zafón ${ }^{15}$, Eva Sanz ${ }^{15}$, \\ Amelia Oleaga ${ }^{8}$, Orosia Bandrés ${ }^{16}$, Sergio Donnay ${ }^{17}$, Ana Megía ${ }^{18}$, María Picallo ${ }^{9}$, \\ Cecilia Sánchez Ragnarsson ${ }^{7}$, Gloria Baena-Nieto ${ }^{19}$, José Carlos Fernández-García ${ }^{20}$, \\ Beatriz Lecumberri ${ }^{6}$, Manel Sahún de la Vega ${ }^{21}$, Ana R. Romero-Lluch ${ }^{14}$, Pedro Iglesias ${ }^{1,2}$
}

${ }^{1}$ Department of Endocrinology, Hospital Universitario Ramón y Cajal, Madrid, Spain; ${ }^{2}$ Present address: Department of Endocrinology, Hospital Universitario Puerta de Hierro Majadahonda, Instituto de Investigación Sanitaria Puerta de Hierro Segovia de Arana, Madrid, Spain; ${ }^{3}$ Department of Endocrinology, Complejo Hospitalario de Navarra, Pamplona, Spain; ${ }^{4}$ Department of Endocrinology, Complejo Hospitalario de Toledo, Toledo, Spain; ${ }^{5}$ Department of Endocrinology, Complejo Asistencial Universitario de León, León, Spain; ${ }^{6}$ Department of Endocrinology, Hospital Universitario La Paz, Madrid, Spain; ${ }^{7}$ Department of Endocrinology, Hospital Universitario Central de Asturias, Oviedo, Spain; ${ }^{8}$ Department of Endocrinology, Hospital Universitario de Basurto, Bilbao, Spain; ${ }^{9}$ Department of Endocrinology, Hospital Universitario Gregorio Marañón, Madrid, Spain; ${ }^{10}$ Department of Endocrinology, Complejo Hospitalario de Jaén, Jaén, Spain; ${ }^{11}$ Department of Endocrinology, Hospital Universitario Virgen de las Nieves, Granada, Spain; ${ }^{12}$ Department of Endocrinology, Hospital Universitario Príncipe de Asturias, Alcalá de Henares, Madrid, Spain; ${ }^{13}$ Department of Endocrinology, Clínica Universidad de Navarra, Pamplona, Spain; ${ }^{14}$ Department of Endocrinology, Hospital Universitario Virgen del Rocío, Sevilla, Spain; ${ }^{15}$ Department of Endocrinology, Hospital Universitario Vall d'Hebron, Barcelona, Spain; ${ }^{16}$ Department of Endocrinology, Hospital Royo Villanova, Zaragoza, Spain; ${ }^{17}$ Department of Endocrinology, Fundación Hospital de Alcorcón, Alcorcón, Madrid, Spain; ${ }^{18}$ Department of Endocrinology, Hospital Universitari de Tarragona Joan XXIII, IISPV, Universitat Rovira i Virgili, Ciberdem, Tarragona, Spain; ${ }^{19}$ Department of Endocrinology, Hospital de Jerez, Jerez de la Frontera, Cádiz, Spain; ${ }^{20}$ Department of Endocrinology, Hospital Universitario Virgen de la Victoria, Málaga, Spain; ${ }^{21}$ Department of Endocrinology, Hospital Moisès Broggi, Sant Joan Despí, Barcelona, Spain

Contributions: (I) Conception and design: JJ Díez, P Iglesias; (II) Administrative support: All authors; (III) Provision of study materials or patients: All authors; (IV) Collection and assembly of data All authors; (V) Data analysis and interpretation: All authors; (VI) Manuscript writing: All authors; (VII) Final approval of manuscript: All authors.

Correspondence to: Juan J. Díez. Department of Endocrinology, Hospital Universitario Puerta de Hierro Majadahonda, Instituto de Investigación Sanitaria Puerta de Hierro Segovia de Arana, Calle Manuel de Falla, 1, 28222 Majadahonda, Madrid, Spain. Email: juanjose.diez@salud.madrid.org.

Background: Recent guidelines for the treatment of hypoparathyroidism emphasize the need for long-term disease control, avoiding symptoms and hypocalcaemia. Our aim has been to analyze the prevalence of poor disease control in a national cohort of patients with hypoparathyroidism, as well as to evaluate predictive variables of inadequate disease control.

Methods: From a nation-wide observational study including a cohort of 1792 patients undergoing total thyroidectomy, we selected 260 subjects [207 women and 53 men, aged (mean \pm SD) $47.2 \pm 14.8$ years] diagnosed with permanent hypoparathyroidism. In every patient demographic data and details on surgical procedure, histopathology, calcium $(\mathrm{Ca})$ metabolism, and therapy with $\mathrm{Ca}$ and calcitriol were retrospectively collected. A patient was considered not adequately controlled (NAC) if presented symptoms of hypocalcemia or biochemical data showing low serum Ca levels or high urinary Ca excretion.

Results: Two hundred and twenty-one (85.0\%) patients were adequately controlled (AC) and 39 (15.0\%) were NAC. Comparison between AC and NAC patients did not show any significant difference in demographic, surgical, and pathological features. Rate of hospitalization during follow-up was significantly higher among NAC patients in comparison with AC patients (35.9\% vs. $10.9 \%, \mathrm{P}<0.001)$. Dose of oral 
$\mathrm{Ca}$ and calcitriol were also significantly higher in NAC subjects. In a subgroup of 129 patients with serum parathyroid hormone (PTH) levels available, we found that NAC patients exhibited significantly lower postoperative $\mathrm{PTH}$ concentrations than AC patients [median (interquartile range) 3 (1.9-7.8) vs. 6.9 (3.0-11) $\mathrm{pg} / \mathrm{mL} ; \mathrm{P}=0.009]$.

Conclusions: In a nation-wide cohort of 260 subjects with definitive hypoparathyroidism, $15 \%$ of them had poor disease control. These patients required higher doses of oral $\mathrm{Ca}$ and calcitriol, had higher rate of hospitalization during follow-up and showed lower PTH concentrations in the postoperative period.

Keywords: Permanent hypoparathyroidism; adequacy of control; thyroidectomy; parathyroid hormone (PTH)

Submitted Feb 27, 2020. Accepted for publication Jul 15, 2020.

doi: $10.21037 /$ gs-20-288

View this article at: http://dx.doi.org/10.21037/gs-20-288

\section{Introduction}

Hypoparathyroidism is defined by low serum calcium $(\mathrm{Ca})$ and inappropriately low or insufficient circulating parathyroid hormone (PTH) levels $(1,2)$. Definitive or permanent hypoparathyroidism is the most common chronic complication of total thyroidectomy (3). From a clinical point of view, it may be defined as the need for long-term $\mathrm{Ca}$ and vitamin $\mathrm{D}$ supplements to avoid clinical or analytical hypocalcemia after surgery. There is lack of consensus regarding the follow-up time after surgery necessary to diagnose definitive hypoparathyroidism. Some authors consider that 6 months $(1,2)$ are sufficient, while others extend this time period to one year (4).

The clinical significance of this hormonal disorder derives, firstly, from its chronic nature, which implies the need for life-long replacement therapy and, secondly, from the risk of deterioration of health-related quality of life $(5,6)$ and of the development of complications and comorbidities (7).

Recent clinical guidelines point to disease control objectives in order to avoid the risk of complications. Among these objectives are the relief of symptoms and achieving normal levels of serum $\mathrm{Ca}$ and phosphorus (P), a CaxP product below $55 \mathrm{mg}^{2} / \mathrm{dL}^{2}$, and avoiding hypercalciuria (2). However, it is not easy to achieve these goals in all patients. A recent report showed that $28 \%$ of patients with chronic hypoparathyroidism were not adequately controlled (NAC) according to criteria established in guidelines (8). In consequence, finding clinical or analytical variables that can predict the development of poor disease control is of clinical interest for the prevention of chronic complications.

In a recent multicentric and nation-wide study in patients undergoing total thyroidectomy, we reported that the prevalence of permanent hypoparathyroidism in clinical practice in Spain was $14.5 \%$, and we also evaluated clinical and analytical variables that were predictors for definitive hypoparathyroidism (9). Our purpose in the present study was to assess the prevalence of poor disease control in this national cohort of patients with hypoparathyroidism, as well as to analyze the clinical, surgical, pathological, and biochemical variables associated to inadequate disease control. We present the following article in accordance with the STROBE reporting checklist (available at http://dx.doi. org/10.21037/gs-20-288).

\section{Methods}

\section{Study design}

This study was conducted in accordance with the Declaration of Helsinki (as revised in 2013). The study was approved by the board of directors of the Sociedad Española de Endocrinología y Nutrición and the ethics committee of the Hospital Universitario Ramón y Cajal (Madrid) (Acta 328, 27/07/2017). Since this is a non-intervention and exclusively retrospective analysis of data from routine clinical practice, the informed consent of the patients has not been considered necessary. All the details on the patients and methodology used are described in detail in a previous report (9). Briefly, this is a retrospective observational study in which each researcher recruited all patients submitted to total thyroidectomy who attended their outpatient clinic between January 1 and March 31, 2018. Inclusion criteria were age $>14$ years at the time of thyroidectomy, availability of surgical and pathological reports, and follow-up in the same hospital for at least one year after thyroidectomy. 
In every patient demographic data, details on surgical procedure, histopathological data, analytical data on Ca metabolism, and information on therapy with $\mathrm{Ca}$ and calcitriol were retrospectively collected. All investigators retrieved clinical information at various stages, i.e., at discharge from hospital after surgery, 3-6 months after surgery, 12 months after the surgery, and at last visit.

\section{Patients}

Of the 1,792 patients undergoing total thyroidectomy, hypoparathyroidism was diagnosed at discharge from surgery in 866 . Of these, a group of 260 presented hypoparathyroidism at the last visit and were diagnosed with permanent hypoparathyroidism (Figure 1). In this group of hypoparathyroid patients there were 207 women (79.6\%) and 53 men $(20.4 \%)$, with a mean age $( \pm$ SD) of $47.2 \pm 14.8$ years and a median [interquartile range (IQR)] follow-up time of 58 (28.5-109.5) months. One-stage thyroidectomy was performed in 234 patients $(90.0 \%)$, whereas 26 subjects $(10.0 \%)$ underwent 2 -stage thyroidectomy. Histopathological results showed benign thyroid disease in 70 subjects $(26.9 \%)$ and thyroid cancer in 190 subjects (73.1\%).

In this cohort of studied patients, researchers recorded the need for at least one hypoparathyroidism-related hospitalization during follow-up, the need for treatment with $\mathrm{Ca}$ and calcitriol, as well as the doses of these two agents. The researchers also rated the adequacy of disease control. A patient was considered to be NAC if he or she had at least one of the following criteria at last visit: serum $\mathrm{Ca}<8 \mathrm{mg} / \mathrm{dL}$, hypercalciuria ( $>300 \mathrm{mg} / 24 \mathrm{~h}$ in females or $>400 \mathrm{mg} / 24 \mathrm{~h}$ in males), and the presence of signs or symptoms of hypocalcemia. To avoid bias as much as possible, the researchers tried to collect data from the medical records as completely as possible.

\section{Statistical analysis}

For quantitative variables, results are expressed as mean \pm SD for normally distributed data and as median (IQR) for nonparametric data. Adjustment to normal distribution was tested by the Kolmogorov test. Categorical variables are described as ratios or percentages. For comparisons of means between two groups of patients, the Student's $t$-test was used for normally distributed data, and the Mann-Whitney U-test was used for nonparametric data. For ratio comparisons, the chi-square test or Fisher's exact test was used. Several models of logistic regression analysis were used to assess the adequacy of the control of hypoparathyroidism as a function of quantitative and qualitative variables. Since not all the variables were available in all the patients in this retrospective study, we have indicated both in the text and tables, the sample size of each variable when it was not the maximum possible. In the logistic regression analysis, we have used several models with different independent variables and with an indication of the sample size in each case. All used tests were two-sided and differences were considered significant when $\mathrm{P}<0.05$.

\section{Results}

Of the cohort of 260 subjects with permanent hypoparathyroidism, there were $221(85.0 \%)$ adequately controlled (AC) and 39 (15.0\%) NAC patients (Figure 1). The causes of inadequacy were: serum $\mathrm{Ca}<8 \mathrm{mg} / \mathrm{dL}$ in 21 patients, hypercalciuria in 10 patients and the presence of symptoms in 14 patients. Thirty-three patients had only one of these criteria, while 6 patients exhibited 2 criteria.

When AC and NAC patients were compared, we could not find significant differences in demographic (gender, age, time of follow-up), surgical (specialized surgical team, extent of surgery, type of thyroidectomy, surgical complications, identification of parathyroid glands and autotransplantation), pathological (parathyroid tissue at histology, thyroid histopathology), and analytical features (postoperative corrected Ca) (Table 1). However, the proportion of patients who needed hypoparathyroidismrelated hospitalization during follow-up was significantly higher in the group of NAC patients $(35.9 \%$ vs. $10.9 \%$, $\mathrm{P}<0.001)$. In addition, doses of oral $\mathrm{Ca}(\mathrm{P}<0.05)$ and calcitriol $(\mathrm{P}<0.01)$ were significantly higher in the group of NAC subjects.

We further analyzed a subgroup of 129 patients in which serum PTH in the immediate postoperative period were available. This subgroup consisted of 97 women $(75.2 \%)$ and 32 men $(24.8 \%)$, aged $47.0 \pm 15.1$ years and with a follow-up time of 46 (23.0-87) months. Serum postoperative PTH concentrations were significantly lower in NAC patients in comparison with AC patients [3 (1.9-7.8) vs. $6.9(3.0-11) \mathrm{pg} / \mathrm{mL} ; \mathrm{P}=0.009$; Figure 2].

Several models of logistic regression analysis were performed to study the dependence of the variable adequacy of disease control as a function of several independent variables, including PTH, demographic, surgical, pathological, follow-up and analytical features 


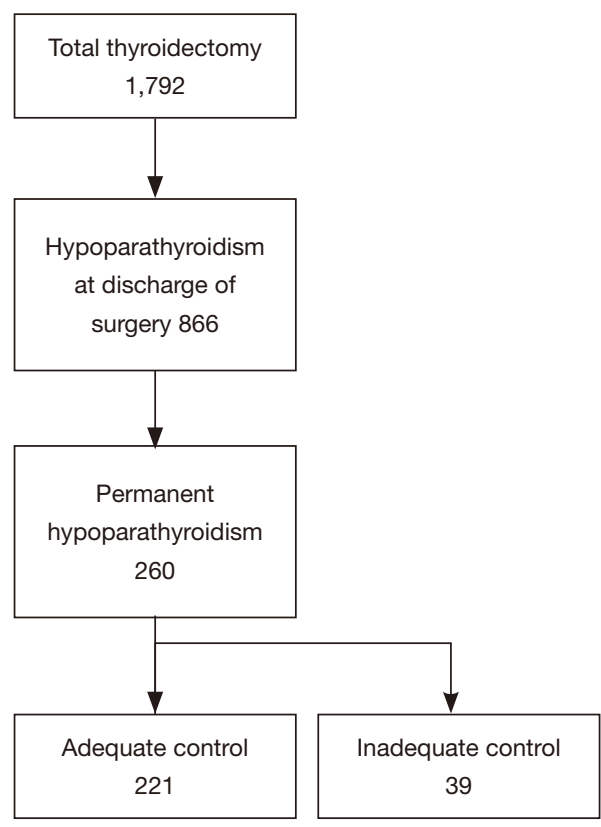

Figure 1 Flow chart of the investigated cohort of patients with total thyroidectomy.

(Table 2). The results showed that inadequate control of hypoparathyroidism was negatively and significantly related to postoperative serum PTH both in the univariate analysis and in different multivariate models. We could not find any significant relationship between the adequacy of the control of hypoparathyroidism and any of the demographic, surgical, pathological, follow-up and analytical features, except for serum PTH.

\section{Discussion}

The results of the present study show that $15 \%$ of our nation-wide cohort of 260 patients with permanent hypoparathyroidism have poor disease control. Most NAC patients exhibited low Ca concentrations, but $25.6 \%$ of them showed hypercalciuria and $35.9 \%$ had symptoms of hypocalcemia. Several studies on the prevalence of permanent hypoparathyroidism after thyroidectomy have been recently reported (10-13). Nevertheless, only a few studies report the percentage of AC and NAC patients and, to the best of our knowledge, none has analyzed the risk factors for poor long-term disease control.

It is of note that biochemical alterations are common in patients with hypoparathyroidism. Marucci et al. (14) assessed biochemical data in 509 patients with hypoparathyroidism, and reported that $43.8 \%$ of patients had low Ca levels, and that hypercalciuria was exhibited by $39.2 \%$ of males and $38.9 \%$ of females. A recent retrospective study in 614 patients with hypoparathyroidism showed that 172 of them (28\%) were NAC according to guideline-based definition, that is, persistent symptoms, serum Ca levels $<8.0 \mathrm{mg} / \mathrm{dL}$, serum P levels $>4.5 \mathrm{mg} / \mathrm{dL}$, or hypercalciuria $(>300 \mathrm{mg}$ /day in males or $>250 \mathrm{mg} /$ day in females) (8). Interestingly, the percentage of NAC patients according to the assessment of the physician in this study was only $11 \%$. This suggests that the subjective assessment of the physician may fail to detect inadequate control. Our study was retrospective and mainly dependent on the individual assessment of each participating physician. Hence, it is possible that our data underestimate the actual percentage of NAC patients.

Our data (Table 2) show that there is higher proportion of NAC patients in females, in patients with 2-stage thyroidectomy, with parathyroid tissue at histology and operated by non-expert surgical teams, thus suggesting that poor control is related to surgical action. However, statistical analysis showed that the adequacy of disease control was not related to the age or sex of the patients, nor to any of the clinical, surgical or histological characteristics analyzed. There was a significant relationship between the control of permanent hypoparathyroidism and the postoperative levels of PTH. Patients with lower concentrations of PTH in the immediate postoperative period seem to be more likely to have poor long-term disease control. This finding was verified in multivariate regression analysis, even when the postoperative $\mathrm{Ca}$ was added to the analysis, implying a sample size reduction.

PTH after thyroidectomy has been related to recovery of parathyroid function, and low postoperative PTH has been reported to be associated with permanent hypoparathyroidism (10,15-19). Furthermore, it is known that the number of parathyroid glands remaining in situ, and therefore the number of PTH producing cells, is a predictor of parathyroid function recovery $(20,21)$. On the contrary, the presence of parathyroid glands in the specimen, i.e., inadvertent removal of parathyroid glands, and therefore removal of PTH producing cells, has been significantly related to permanent hypoparathyroidism $(9,13,22)$. However, explaining how the postoperative concentration of PTH can influence long-term disease control is not easy. One possible explanation is that patients with higher levels of postoperative PTH have a greater parathyroid functional reserve or a greater capacity of the 
Table 1 Demographic, surgical, pathological, analytical and follow-up features of studied patients with permanent hypoparathyroidism

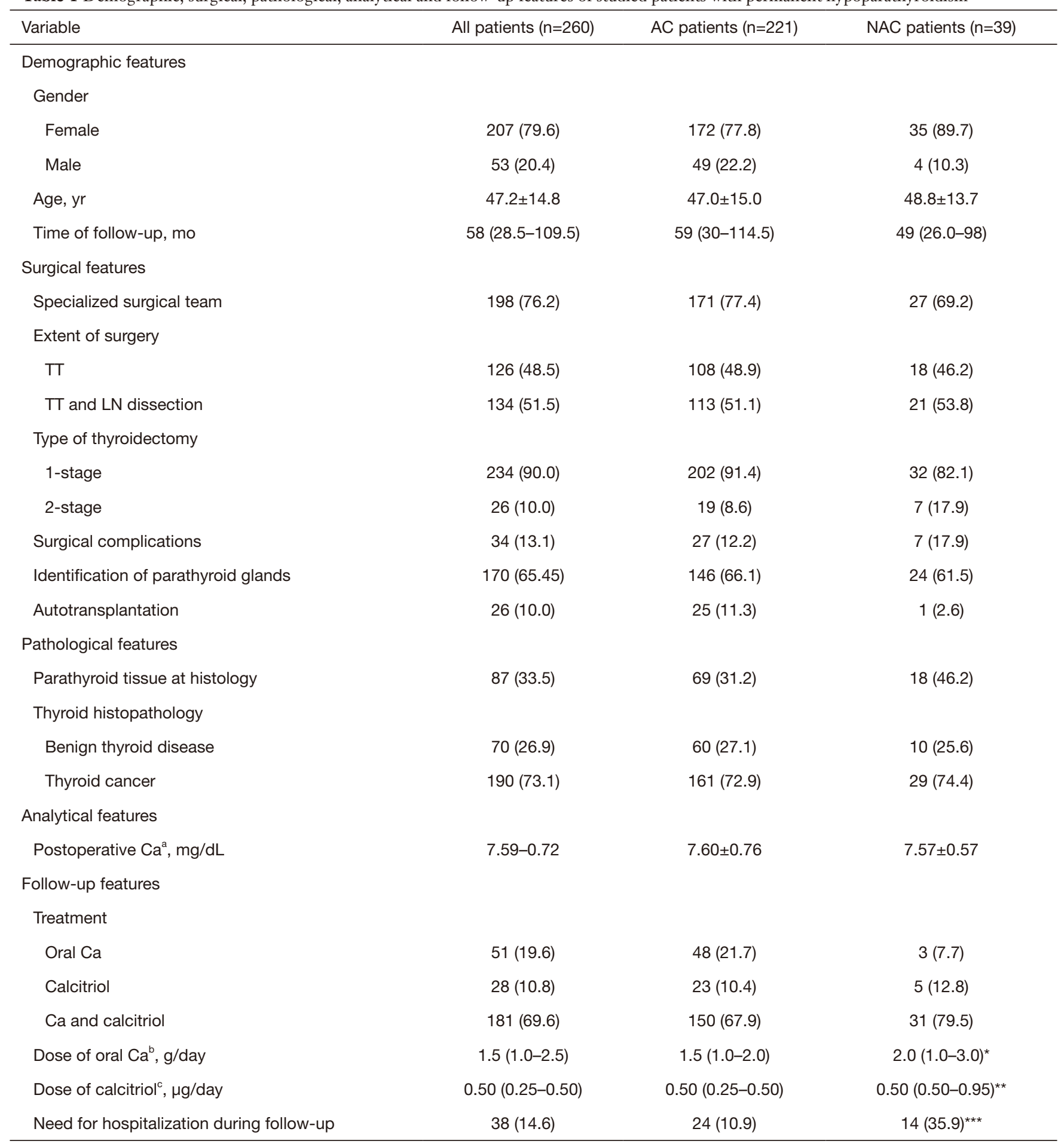

Data are the number of patients (percentage), mean \pm SD for normally distributed data and median (interquartile range) for nonparametric data. ${ }^{*} \mathrm{P}<0.05$; ${ }^{*} \mathrm{P}<0.01$; ${ }^{* \star *} \mathrm{P}<0.001$. ${ }^{\mathrm{a}} \mathrm{n}=188$ (153 adequate control, 35 inadequate control); ${ }^{\mathrm{b}} \mathrm{n}=225$ (191 adequate control, 34 inadequate control); ${ }^{c} n=204$ (168 adequate control, 36 inadequate control). AC, adequately controlled, NAC, not adequately controlled; TT, total thyroidectomy; LN, lymph node; Ca, calcium. 


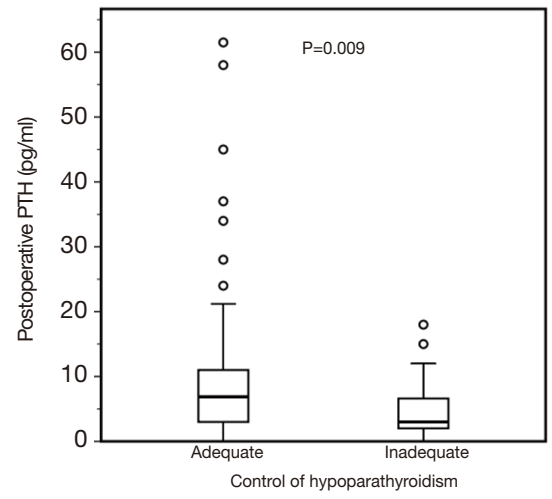

Figure 2 Box plot diagrams showing postoperative parathyroid hormone (PTH) concentrations in patients with permanent hypoparathyroidism classified according the control of the disease (adequate, inadequate). The bottom and top of the box indicate the lower (Q1) and upper (Q3) quartiles, respectively; median is the band near the middle of the box. The bottom and top 'whiskers' are the smallest and largest nonoutliers observations, respectively. Circles represent outliers (>1.5-fold interquartile range, IQR = Q3-Q1).

Table 2 Results of four models of logistic regression to study the influence of postoperative serum PTH concentrations (pg/mL) on the presence of inadequacy of the control of permanent hypoparathyroidism

\begin{tabular}{|c|c|c|c|c|c|c|}
\hline Model & Independent variables included in the model & $\mathrm{n}$ & $\begin{array}{c}\text { Significant variables } \\
\text { in the model }\end{array}$ & OR & $95 \% \mathrm{Cl}$ & $P$ \\
\hline 1 & PTH (univariate) & 129 & PTH & 0.91 & $0.83-0.99$ & 0.041 \\
\hline 3 & PTH, demographic and surgical features & 129 & PTH & 0.88 & $0.79-0.98$ & 0.022 \\
\hline 6 & PTH, demographic, surgical, pathological and analytical features & 104 & PTH & 0.84 & $0.75-0.96$ & 0.007 \\
\hline 7 & $\begin{array}{l}\text { PTH, demographic, surgical, pathological, follow-up and analytical } \\
\text { features }\end{array}$ & 76 & PTH & 0.81 & $0.68-0.96$ & 0.015 \\
\hline
\end{tabular}

Demographic features: gender, age and time of follow-up (months). Surgical features: specialized surgical team, extent of surgery, type of thyroidectomy, surgical complications, identification of parathyroid glands and autotransplantation. Pathological features: parathyroid tissue at histology, thyroid histopathology. Follow-up features: dose of oral Ca (g/day), dose of calcitriol ( $\mu \mathrm{g} / \mathrm{day}$ ) and need for hospitalization during follow-up. Analytical features: postoperative corrected $\mathrm{Ca}$ (mg/dL). OR, odds ratio; $\mathrm{Cl}$, confidence interval; PTH, parathyroid hormone; Ca, calcium.

parathyroid glands to partially recover their function and, therefore, make the control of hypoparathyroidism with $\mathrm{Ca}$ and vitamin D more easily attainable.

It is well known that patients with permanent hypoparathyroidism have an increased risk of renal stones and renal failure, and that this may be linked to prolonged use of $\mathrm{Ca}$ and calcitriol (23). Interestingly, we observed that our NAC patients required higher doses of $\mathrm{Ca}$ and calcitriol. A higher dose of calcitriol has been linked with the development of hypercalciuria and with renal function loss (24). In another study, renal failure and poorer control of serum Ca levels were more common in patients treated with calcitriol than in those treated with calcifediol (25).

In addition, in recent years, various epidemiological studies have shown that chronic postsurgical hypoparathyroidism increases the risk of several conditions, including ischemic cardiovascular disease $(22,26)$, neuropsychiatric disease $(26,27)$, seizures $(26,27)$, cataract $(28)$, and mortality $(28,29)$. Biochemical findings such as those found in NAC patients seem to be related with these complications. The analysis 
by Underbjerg et al. (30) clearly established that lower timeweighted serum $\mathrm{Ca}$ was related to the risk of cardiovascular disease and high time-weighted $\mathrm{P}$ was related with mortality and risk of infection. The number of episodes of hypercalcemia and the duration of the disease was related to mortality, risk of infections, cardiovascular and renal disease.

Our NAC patients required hospitalization during follow-up more frequently than AC patients. This is in agreement with studies that demonstrated that clinical burden and healthcare resource utilization among NAC patients are higher than those in patients with adequate disease control. The study by Chen et al. (8) showed that the number of outpatient visits, emergency room visits, and hospitalization was significantly higher in NAC patients than in comparison with AC subjects.

As a retrospective survey, our study has limitations. We acknowledge that different laboratory procedures have been employed to measure $\mathrm{Ca}$ and $\mathrm{PTH}$, and that there is a lack of common protocols in all participating centers. Each researcher carried out periodic visits of the patients according to their judgment and there was no common criterion about the frequency of $\mathrm{Ca}$ measurements in blood and urine. Our definition of NAC patient is not accurate, since not all patients had complete biochemical data, but it is similar to that used by other authors in clinical studies (31). It is possible that there are more cases of hypercalciuria than those detected in this retrospective study. Furthermore, we had no data on serum phosphorus, magnesium and 25-hydroxyvitamin D. Data on serum PTH to assess the parathyroid functional reserve at last visit were also not available. Main strengths of our study are the relatively high sample size, its multicentric and nation-wide nature, and the fact that our design reflects the practice in real-life in Spain.

\section{Conclusions}

In summary, $15 \%$ of patients in a nation-wide cohort of 260 subjects with definitive hypoparathyroidism have poor disease control. Our data suggest that, in this cohort of Spanish patients recruited in real-life clinical practice by endocrinologists, low concentrations of PTH in the postoperative period, reflecting a low parathyroid reserve, may be related and predict a poor long-term disease control. Cohort studies of patients from other countries, as well as prospective studies are needed to confirm and generalize these findings.

\section{Acknowledgements}

Funding: None.

\section{Footnote}

Reporting Checklist: The authors have completed the STROBE reporting checklist. Available at http://dx.doi. org/10.21037/gs-20-288

Data Sharing Statement: Available at http://dx.doi. org/10.21037/gs-20-288

Conflicts of Interest: All authors have completed the ICMJE uniform disclosure form (available at http://dx.doi. org/10.21037/gs-20-288). The authors have no conflicts of interest to declare.

Ethical Statement: The authors are accountable for all aspects of the work in ensuring that questions related to the accuracy or integrity of any part of the work are appropriately investigated and resolved. This study was conducted in accordance with the Declaration of Helsinki (as revised in 2013). The study was approved by the board of directors of the Sociedad Española de Endocrinología y Nutrición and the ethics committee of the Hospital Universitario Ramón y Cajal (Madrid) (ACTA 328, 27/07/2017). Since this is a non-intervention and exclusively retrospective analysis of data from routine clinical practice, the informed consent of the patients has not been considered necessary.

Open Access Statement: This is an Open Access article distributed in accordance with the Creative Commons Attribution-NonCommercial-NoDerivs 4.0 International License (CC BY-NC-ND 4.0), which permits the noncommercial replication and distribution of the article with the strict proviso that no changes or edits are made and the original work is properly cited (including links to both the formal publication through the relevant DOI and the license). See: https://creativecommons.org/licenses/by-nc-nd/4.0/.

\section{References}

1. Bollerslev J, Rejnmark L, Marcocci C, et al. European Society of Endocrinology. European society of endocrinology clinical guideline: Treatment of chronic hypoparathyroidism in adults. Eur J Endocrinol 
2015;173:G1-20.

2. Brandi ML, Bilezikian JP, Shoback D, et al. Management of hypoparathyroidism: Summary statement and guidelines. J Clin Endocrinol Metab 2016;101:2273-83.

3. Orloff LA, Wiseman SM, Bernet VJ, et al. American Thyroid Association statement on postoperative hypoparathyroidism: diagnosis, prevention, and management in adults. Thyroid 2018;28:830-41.

4. Stack BC Jr, Bimston DN, Bodenner DL, et al. American Association of Clinical Endocrinologists and American College of Endocrinology disease state clinical review: postoperative hypoparathyroidism - Definitions and management. Endocr Pract 2015;21:674-85.

5. Hadker N, Egan J, Sanders J, et al. Understanding the burden of illness associated with hypoparathyroidism reported among patients in the PARADOX study. Endocr Pract 2014;20:671-9.

6. Büttner M, Musholt TJ, Singer S. Quality of life in patients with hypoparathyroidism receiving standard treatment: a systematic review. Endocrine 2017;58:14-20.

7. Bollerslev J, Schalin-Jäntti C, Rejnmark L, et al. Unmet therapeutic, educational and scientific needs in parathyroid disorders. Eur J Endocrinol 2019;181:P1-19.

8. Chen K, Krasner A, Li N, et al. Clinical burden and healthcare resource utilization among patients with chronic hypoparathyroidism, overall and by adequately vs. not adequately controlled disease: a multi-country chart review. J Med Econ 2019;22:1141-52.

9. Díez JJ, Anda E, Sastre J, et al. Prevalence and risk factors for hypoparathyroidism following total thyroidectomy in Spain: a multicentric and nation-wide retrospective analysis. Endocrine 2019;66:405-15.

10. Sitges-Serra A, Ruiz S, Girvent M, et al. Outcome of protracted hypoparathyroidism after total thyroidectomy. Br J Surg 2010;97:1687-95.

11. Puzziello A, Rosato L, Innaro N, et al. Hypocalcemia following thyroid surgery: incidence and risk factors. A longitudinal multicenter study comprising 2,631 patients. Endocrine 2014;47:537-42.

12. Edafe O, Antakia R, Laskar N, et al. Systematic review and meta-analysis of predictors of post-thyroidectomy hipocalcemia. Br J Surg 2014;101:307-20.

13. Ritter K, Elfenbein D, Schneider DF, et al. Hypoparathyroidism after total thyroidectomy: Incidence and resolution. J Surg Res 2015;197:348-53.

14. Marcucci G, Cianferotti L, Parri S, et al. HypoparaNet: a database of chronic hypoparathyroidism based on expert medical-surgical centers in Italy. Calcif Tissue Int
2018;103:151-63.

15. Lang BH, Yih PC, Ng KK. A prospective evaluation of quick intraoperative parathyroid hormone assay at the time of skin closure in predicting clinically relevant hypocalcemia after thyroidectomy. World J Surg 2012;36:1300-6.

16. Almquist $M$, Hallgrimsson $P$, Nordenström E, et al. Prediction of permanent hypoparathyroidism after total thyroidectomy. World J Surg 2014;38:2613-20.

17. Hermann M, Ott J, Promberger R, et al. Kinetics of serum parathyroid hormone during and after thyroid surgery. $\mathrm{Br}$ J Surg 2008;95:1480-7.

18. Sitges-Serra A, Gómez J, Barczynski M, et al. A nomogram to predict the likelihood of permanent hypoparathyroidism after total thyroidectomy based on delayed serum calcium and iPTH measurements. Gland Surg 2017;6:S11-9.

19. Canu GL, Medas F, Longheu A, et al. Correlation between iPTH levels on the first postoperative day after total thyroidectomy and permanent hypoparathyroidism: our experience. Open Med (Wars) 2019;14:437-42.

20. Lorente-Poch L, Sancho JJ, Ruiz S, Sitges-Serra A. Importance of in situ preservation of parathyroid glands during total thyroidectomy. Br J Surg 2015;102:359-67.

21. Villarroya-Marquina I, Sancho J, Lorente-Poch L, et al. Time to parathyroid function recovery in patients with protracted hypoparathyroidism after total thyroidectomy. Eur J Endocrinol 2018;178:103-11.

22. Paek SH, Lee YM, Min SY, et al. Risk factors of hypoparathyroidism following total thyroidectomy for thyroid cancer. World J Surg 2013;37:94-101.

23. Underbjerg L, Sikjaer T, Mosekilde L, et al. Cardiovascular and renal complications to postsurgical hypoparathyroidism: a Danish nationwide controlled historic follow-up study. J Bone Miner Res 2013;28:2277-85.

24. Coudenys E, Van Meerhaeghe T, Unuane D, et al. Long-term treatment with calcitriol in postsurgical hypoparathyroidism leads to renal function decline. Horm Metab Res 2019;51:362-6.

25. Lorente-Poch L, Sancho JJ, Carballo L, et al. Clinical profile and long-term follow-up of 32 patients with postoperative permanent hypoparathyroidism. Gland Surg 2017;6:S3-10.

26. Underbjerg L, Sikjaer T, Mosekilde L, et al. The epidemiology of nonsurgical hypoparathyroidism in Dermark: a nationwide case finding study. J Bone Miner Res 2015;30:1738-44.

27. Underbjerg L, Sikjaer T, Mosekilde L, et al. Postsurgical 
hypoparathyroidism-risk of fractures, psychiatric disease, cancer, cataract, and infections. J Bone Miner Res 2014;29:2504-10.

28. Vadiveloo T, Donnan PT, Leese CJ, et al. Increased mortality and morbidity in patients with chronic hypoparathyroidism: a population-based study. Clin Endocrinol (Oxf) 2019;90:285-92.

29. Almquist M, Ivarsson K, Nordenström E, et al. Mortality in patients with permanent hypoparathyroidism after total

Cite this article as: Díez JJ, Anda E, Sastre J, Pérez Corral B, Álvarez-Escolá C, Manjón L, Paja M, Sambo M, Santiago Fernández P, Blanco Carrera C, Galofré JC, Navarro E, Zafón C, Sanz E, Oleaga A, Bandrés O, Donnay S, Megía A, Picallo M, Sánchez Ragnarsson C, Baena-Nieto G, Fernández-García JC, Lecumberri B, Sahún de la Vega M, Romero-Lluch AR, Iglesias P. Permanent postoperative hypoparathyroidism: an analysis of prevalence and predictive factors for adequacy of control in a cohort of 260 patients. Gland Surg 2020;9(5):13801388. doi: $10.21037 /$ gs-20-288 thyroidectomy. Br J Surg 2018;105:1313-8.

30. Underbjerg L, Sikjaer T, Rejnmark L. Long-term complications in patients with hypoparathyroidism evaluated by biochemical findings: a case-control study. J Bone Miner Res 2018;33:822-31.

31. Siggelkow H, Clarke BL, Germak J, et al. Burden of illness in not adequately controlled chronic hypoparathyroidism: findings from a 13-country patient and caregiver survey. Clin Endocrinol (Oxf) 2020;92:159-68. 